\section{Psoriasis: Biologika vor OP doch nicht absetzen?}

\begin{abstract}
Haben Psoriasispatienten, die Biologika einnehmen, tatsächlich mehr postoperative Komplikationen, wenn sie trotz Eingriff die Medikamenteneinnahme fortführen? Eher nicht, wie eine aktuelle britische Studie ergab.
\end{abstract}

\begin{abstract}
A us Angst vor einem höheren Infektionsrisiko und Wundheilungsstörungen lautet aktuell die Empfehlung, eine Biologikatherapie vor einem geplanten größeren chirurgischen Eingriff zu unterbrechen. Daten mit ausreichend hoher Evidenz gibt es allerdings bislang kaum. Britische Dermatologen haben daher anhand von Patientendaten untersucht, inwieweit diese Empfehlung befolgt wird, und gleichzeitig das postoperative Komplikationsrisiko bei Fortführen der Behandlung unter die Lupe genommen. In den Jahren 2005 bis 2011 waren 300 Patienten mit Psoriasis oder Psoriasis-Arthritis (PsA) registriert worden, von denen sich 42 insgesamt 77-mal operieren lassen mussten. Die Eingriffe umfassten 40 kleinere Operationen überwiegend an der Haut und 37 größere. Nur in 20 Fällen war die Behandlung unterbrochen worden. Ausgesetzt wurde die Therapie vor allem bei kardio-
\end{abstract}

thorakalen $(66,6 \%)$ und orthopädischen (40\%) Operationen, seltener bei Eingriffen an der Haut (17,5\%). Das meistverschriebene Biologikum war Etanercept (30 Eingriffe) gefolgt von Adalimumab (28 Eingriffe) und Infliximab (11 Eingriffe).

In acht Fällen traten postoperative Komplikationen auf, dabei konnten deutlich mehr Komplikationen bei den Operationen mit Behandlungsstopp (25,0\%) registriert werden als bei Eingriffen, bei denen die Biologikaeinnahme fortgeführt worden war (5,2\%). Auch bei Berücksichtigung verschiedener Risikofaktoren, wie begleitende immunsuppressive Therapie, Diabetes und Rauchen, blieb das postoperative Komplikationsrisiko in der Gruppe mit perioperativem Behandlungsstopp signifikant höher als in der Gruppe mit Behandlungsunterbrechung $(\mathrm{p}=0,014)$. Erwartungsgemäß stieg gleichzeitig mit Absetzen das Risiko eines
Wiederaufflammens der Psoriasis oder PsA ( $p=0,0003)$. Da die Leitlinienempfehlung auf größere Eingriffe abzielt, beschränkten die britischen Dermatologen die Analyse auch explizit auf umfangreiche Operationen, ohne dass dabei das postoperative Komplikationsrisiko bei fortgeführter Biologikaeinnahme gestiegen wäre.

Fazit: Ein Fortsetzen der Biologikatherapie bedingt offenbar kein erhöhtes postoperativer Komplikationsrisiko. Im Gegenteil, Patienten, die perioperativ ihr Biologikum abgesetzt hatten, hatten unabhängig von weiteren Risikofaktoren postoperativ deutlich mehr Komplikationen. Gleichzeitig begünstigte ein Behandlungsstopp einen Psoriasisschub. Angesichts der kleinen Fallzahlen und des retrospektiven Studiendesigns mahnen die Studienautoren jedoch, das Ergebnis mit Vorsicht zu interpretieren, speziell in Bezug auf große kardiologische und orthopädische Operationen.

Dr. Dagmar Kraus

Bakkour W et al. The risk of post-operative complications in psoriasis and psoriatic arthritis patients on biologic therapy undergoing surgical procedures. JEADV 2016; 30: 86-91

\title{
Teleangiektasien am ganzen Körper
}

Eine 58-jährige Frau berichtete über intermittierende rektale Blutabgänge in den vergangenen sechs Wochen. Sie litt unter zunehmender Müdigkeit und Atemnot. Aus der früheren Anamnese waren wiederholte Episoden von spontanem Nasenbluten bekannt, die nie richtig abgeklärt worden waren.

Bei der körperlichen Untersuchung zeigten sich Teleangiektasien an der Lippenschleimhaut (Abb. A) und am Nagelfalz (Abb. B). Im Gespräch stellte sich heraus, dass auch die Mutter häufig Nasenbluten hatte und bei ihr ähnliche Hautveränderungen bestanden.

Im Labor zeigte sich eine ausgeprägte Eisenmangelanämie mit einem $\mathrm{Hb}$ von 8,1 $\mathrm{g} / \mathrm{dl}$ und einem mittleren korpuskulären Volumen (MCV) von $72 \mathrm{fl}$. In der Koloskopie stellte mal zahlreiche arteriovenöse Malformationen und Teleangiektasien fest (Abb. C). Pulmonale oder zerebrale Gefäßmalformationen fanden sich im Zuge der weiteren Abklärung nicht.

Anhand der klinischen Befunde stellte man die Diagnose einer hereditären hämorrhagischen Teleangiektasie, die auch als Osler-Weber-Rendu-Syndrom bekannt ist. Eine molekularbiologische Analyse ergab eine Genmutation im Endoglin-Gen bei der Patientin und ihrer Mutter. Damit war die molekulare Diagnose gesichert. Prof. Hermann S. FüeßI

Pérez-Belmonte LM et al. Osler-Weber-Rendu-Syndrome. N Engl J Med 2015; 373:e15

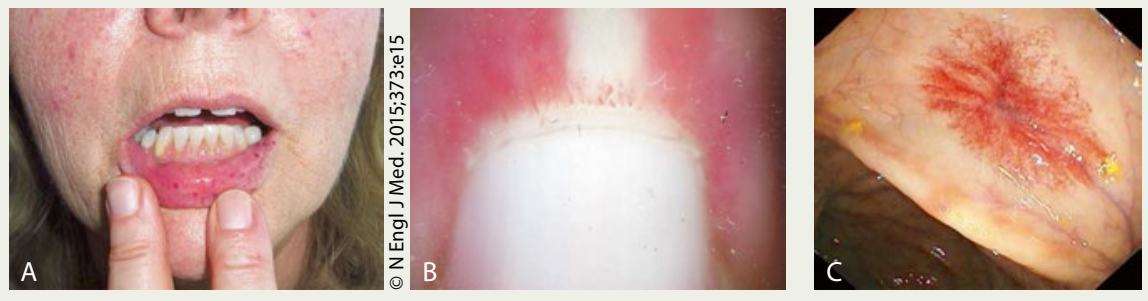

Teleangiektasien an der Lippenschleimhaut (A), am Nagelfalz (B) und im Dickdarm (C). 